\title{
Spherical symmetrical approach to the theory of runaway breakdown
}

Book or Report Section

Accepted Version

Gurevich, A. V., Lukyanov, A. V., Zybin, K. P. and RousselDuprè, R. A. (2002) Spherical symmetrical approach to the theory of runaway breakdown. In: Kortshagen, $\mathrm{U}$. and Tsendin, L. D. (eds.) Electron Kinetics and Applications of Glow Discharges. NATO Science Series: B, 367. Springer, Boston, MA, pp. 19-35. ISBN 9780306470769 doi: https://doi.org/10.1007/0-306-47076-4_2 Available at http://centaur.reading.ac.uk/95162/

It is advisable to refer to the publisher's version if you intend to cite from the work. See Guidance on citing.

To link to this article DOI: http://dx.doi.org/10.1007/0-306-47076-4_2

Publisher: Springer, Boston, MA 
copyright holders. Terms and conditions for use of this material are defined in the End User Agreement.

\section{www.reading.ac.uk/centaur}

\section{CentAUR}

Central Archive at the University of Reading

Reading's research outputs online 


\title{
SPHERICAL SYMMETRICAL APPROACH TO THE THEORY OF RUNAWAY BREAKDOWN
}

\author{
A. V. Gurevich, ${ }^{1}$ A. V. Lukyanov, ${ }^{1}$ K. P. Zybin, ${ }^{1}$ and \\ R. A. Roussel-Duprè 2 \\ ${ }^{1}$ P. N. Lebedev Institute of Physics \\ Russian Academy of Sciences \\ 117924 Moscow, Russia \\ 2 Space and Science Technology Division \\ Los Alamos National Laboratory \\ Los Alamos, NM
}

\section{INTRODUCTION}

The notion that secondary electrons generated by cosmic rays could be accelerated over kilometer distances by thunderstorm electric field was first proposed by Wilson. ${ }^{1}$ The frictional force $F$ of fast nonrelativistic electrons moving in air diminishes with the kinetic energy of electrons $\varepsilon$ (Fig. 1) until a minimum value,

$$
F_{\min } \simeq \frac{4 \pi N_{n} Z e^{4}}{m c^{2}} a, \quad a \simeq 11
$$

is reached.at. $\varepsilon_{\min } \simeq 3-4 m c^{2}$. At relativistic energies $\varepsilon>m c^{2}$ the force $F$ is slowly growing $\left(\sim \ln \left(\varepsilon / \varepsilon_{1}\right), \varepsilon_{1} \simeq 270 \mathrm{eV}\right)$ This specific behavior of friction force $F$ in nonrelativistic region is a cause of runaway effect in a constant electric field. ${ }^{2}$ Clearly, if an electric field $E$, applied to the medium, exceeds the minimum value $F_{\min }$ :

$$
E>E_{c}=F_{\min } / e=\frac{4 \pi N_{m} Z e^{3}}{m c^{2}} a
$$

then the electrons with the energies $\varepsilon$ greater than characteristic value $\varepsilon_{c}=m c^{2}\left(\gamma_{1 c}-1\right)$ (Fig. 1) will runaway. In other words electrons will be accelerated up to very high energies.

Runaway electrons in the context of thunderstorm electric fields in air were studied previously by a number of authors. ${ }^{3,4}$ A fundamental new idea proposed by Gurevich, Milikh and Roussel-Dupree in 1992 showed how the generation of an avalanche of runaway electrons could lead to the air breakdown. ${ }^{5}$ As is well known impact ionization of the air by energetic electrons leads to the production of newborn electrons with a wide spectrum of energies. Those secondary electrons whose energy exceeds the characteristic value $\varepsilon_{c}$ become a part of runaway population and contribute 


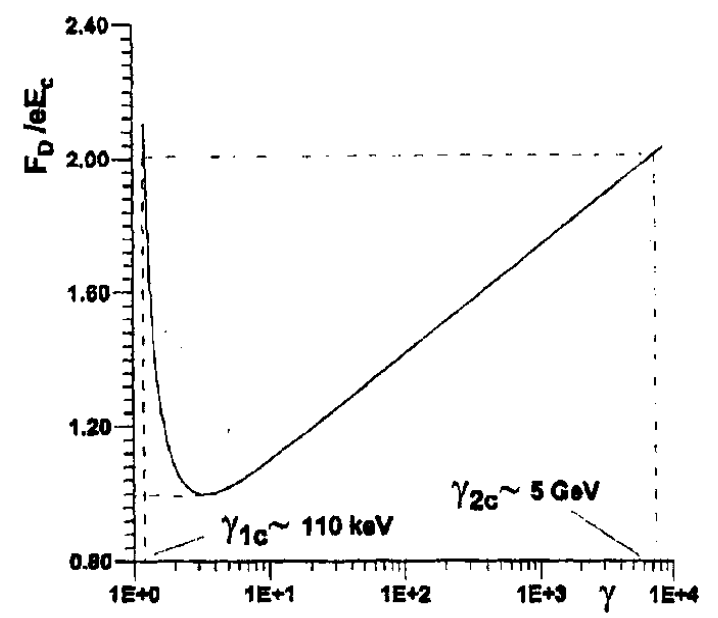

Figure 1. Dynamical friction force. The normalized energy loss per unit length $\left(F_{d} / E c 0\right)$ for electrons in air is plotted as a function of the electron Lorentz factor $\gamma$.

to further acceleration and ionization that again populates the runaway regime. The net result is an avalanche in which the number of runaway electrons and with them of electrons of all other energies grows exponentially. This process was called runaway breakdown. It proceeds in electric fields $E>E_{c}$ (2) and in the presence of highenergy electrons (produced as a results of cosmic-ray interaction with atmosphere). It is important to note, that the critical field for runaway breakdown is about an order of magnitude lower than the threshold field for conventional breakdown $E_{c t h}$ :

$$
E_{c} \approx 0.1 E_{c t h}
$$

This fact makes it possible for runaway breakdown to take place in relatively low electric fields $E_{c} \leq E<<E_{c t h}$ and as a result to determine fast charge transfer process in thundercloud, which can have important implication for lightning preconditioning and overall development of thunderstorm electricity.

Runaway breakdown proceeds as a beam of fast electrons having maximum energies of the order of $1 \mathrm{MeV} .{ }^{6} \mathrm{~A}$ detailed kinetic theory ${ }^{7}$ for the runaway process permitted a precise determination of the intensive fluxes of $\mathrm{X}$-rays produced by bremsstrahlung emission in the $10-500 \mathrm{keV}$ energy range.

The experimental observations of intensive X-rays by McCarthy and Parks ${ }^{8}$ could be considered as a first manifestation of runaway breakdown during thunderstorm. These effects were recently confirmed and studied in details by Eack, Beasley et al.. ${ }^{9-11}$ Even more convincing were the observation of very intensive $\gamma$-ray bursts by' BATSE. ${ }^{12}$ These observations indicate the direct connection between spectacular high-altitude lighting and runaway breakdown.

Nevertheless, it is worthy to note that the Earth's magnetic field B can substantially affect the runaway breakdown process at the altitudes above $z \sim 20 \mathrm{~km}$. At $z \simeq 40 \mathrm{~km}$, this influence already dominates. That is why, the influence of magnetic field $\mathrm{B}$ on high-altitude lighting should turn out to be significant even though it strongly depends on the angle between $\mathrm{E}$ and B. ${ }^{13,14}$

Moreover, the role of lighting emission is not completely understood. For instance, in Refs. 15, 16 is supposed that alternating electromagnetic field is just a source of air ionization growth produced by high altitude lighting at $z \sim 60-80 \mathrm{~km}$. While, in such a case in Refs. 15-17 only conventional breakdown in gas was considered. From the one hand it gives rise to some difficulties in interpretation of the breakdown - the field is usually less than needed for conventional breakdown. From the other hand conventional mechanism of gas breakdown can not explain strong $\gamma$-emission which as usually supposed to appear under high-altitude lightnings. 
It is pertinent to remember in connection with this fact that runaway process of electrons may appear not only in constant electric field. In particularly, runaway of electrons and ions may occur under stochastic Fermi acceleration. This process playing possibly a marked role in injection mechanisms of cosmic ray generation ${ }^{18}$ leads to chaotic acceleration and manifests itself as a substantial distortion of the sphericallysymmetrical part of a distribution function. ${ }^{19}$ In this case, a chaotic (thermal) tail in the distribution function and symmetrically spreading in all directions particle flux are observed. One can say that effective temperature $T=T(\varepsilon)$ depends on the particle energy $\varepsilon$, growing infinitely with increasing of the energy $\varepsilon$. Clearly, this process could be called spherical-symmetrical or "thermal" mechanism of runaway. The process may be evolved effectively not only under the Fermi acceleration mechanism, but also under the action of a constant electric field on a plasma in case of strong electron scattering by ions (i.e. at $Z>>1$, where $Z$ is an ion charge) or by isotropically excited ion-sound plasma oscillations. 20,21

It is natural to expect that the same thermal runaway effect arises in motion of fast electrons in air if chaotic alternative electric field is applied or in constant electric and magnetic fields under strong scattering by nuclei $Z>>1$. In air $Z \simeq 7.25$ (in reality, the effective value $Z_{\text {eff }}$ could be slightly lower because of electron shielding on low levels ${ }^{7}$ ). Undoubtedly, that the value of $Z$ in air is insufficient for total stochastization of electron motion in a constant electric field. In the same time, in the first approximation, even in this case consideration of chaotic acceleration could be useful. It simplifies significantly the kinetic theory of the air breakdown and gives it possible to solve the problem in different conditions: with and without magnetic field, in oscillating electric field a.s.o. However, what are the conditions, when spherical symmetrical (thermal) approach is correct? Can that thermal mechanism of runaway give rise to gas breakdown? Is that process significant in air, i.e. what is the relationship between critical field of thermal mechanism of runaway breakdown in crossed electric and magnetic fields or in oscillating electric field and the critical field of conventional breakdown?

This paper is just devoted to the investigation of these problems.

\section{TRANSFORMATION OF KINETIC EQUATION}

To describe the breakdown on runaway particles in electric field $\mathbf{E}$ and magnetic field $\mathbf{B}$ we start from kinetic equation ${ }^{7}$ :

$$
\frac{\partial f}{\partial t}+\mathbf{v} \boldsymbol{\nabla} f+e \mathbf{E} \frac{\partial f}{\partial \mathbf{p}}+\frac{e}{c}[\mathbf{v}, \mathbf{B}] \frac{\partial f}{\partial \mathbf{p}}=\hat{S}_{1}(f)+\hat{S}_{\text {ion }}(f)
$$

here $\hat{S}_{\text {ion }}$ is an ionization integral which describe the production of new electrons due to collisions with molecules of the air, $\hat{S}_{1}=\hat{S}_{0}+\hat{L}$. The term $\hat{S}_{0}$ describes electron energy loses and the term $\hat{L}$ is a pitch-angle scattering. The classical integrals $\hat{S}_{0}$ and $\hat{L}$ and a model integral $\hat{S}_{\text {ion }}$ were determined in the paper Ref. 7. In arbitrary coordinate system, terms $\hat{S}_{0}$ and $\hat{L}$ could be presented in a form:

$$
\begin{aligned}
& \hat{S}_{0}(f)=\frac{\partial}{\partial \mathbf{p}}\left(\frac{\mathbf{p}}{p} F_{D} f\right) \\
& \hat{L}(f)=\nu(p) p^{2} \frac{\partial}{\partial p_{i}}\left[\left(\dot{\delta}_{i j}-\frac{p_{i} p_{j}}{p^{2}}\right) \frac{\partial f}{\partial p_{j}}\right] .
\end{aligned}
$$


Here

$$
\begin{aligned}
F_{D}= & \frac{4 \pi Z c^{4} n_{m}}{m c^{2}} \frac{\gamma^{2}}{\gamma^{2}-1} \\
& \times\left\{\ln \left[\frac{m c^{2}\left(\gamma^{2}-1\right)^{1 / 2}(\gamma-1)^{1 / 2}}{\sqrt{2} I}\right]-\left[\frac{2}{\gamma}-\frac{1}{\gamma^{2}}\right] \frac{\ln 2}{2}+\frac{1}{2 \gamma^{2}}+\frac{(\gamma-1)^{2}}{16 \gamma^{2}}\right\}
\end{aligned}
$$

is a dynamical friction force,

$$
\nu=\frac{F_{D}(Z / 2+1)}{p \gamma}
$$

is a collision frequency, $I=80.5 \mathrm{eV}$ for air, $N_{m}$ is the molecular density,

$$
\gamma=\left(1 / \sqrt{1-(v / c)^{2}}\right)
$$

$v$ is the electron speed. Note, that here $Z \approx 14.5$, as we denote it as the average charge of air molecules $\mathrm{N}_{2}$ and $\mathrm{O}_{2}$.

There are several characteristic times in the equations (4), (5). One of them is defined by energy losses $t_{E}$ and the other one by the pitch-angle scattering $t_{\theta}$.

$$
t_{E} \approx \frac{p}{F_{D}} \quad t_{\theta} \approx \frac{p \gamma}{F_{D}(Z / 2+1)}
$$

If the effective charge $Z$ is large enough, then depending on the considered energy range it could be different relation between $t_{E}$ and $t_{\theta}$. If

$$
\gamma \ll 1+\frac{Z}{2}
$$

then

$$
t_{\theta} \ll t_{E}
$$

and distribution function in this region should be close to spherical symmetrical due to dominant angular scattering. On the contrary for high energy electrons

$$
\gamma \gg 1+\frac{Z}{2}
$$

angular scattering is weak enough and distribution function is determined mainly by energy losses and angular distribution of a new born electrons.

We will start our analyses, considering the low energy range, where conditions (7) is fulfilled. Introducing a new small parameter of the problem $\in=t_{\theta} / t_{E}$ we rewrite equations (4) and (5) in the form:

$$
\frac{\partial f}{\partial t}+\mathbf{v} \nabla f+e \mathbf{E} \frac{\partial f}{\partial \mathbf{p}}-\hat{S}_{\mathrm{v}}(f)-\hat{S}_{\mathrm{ion}}(f)=\frac{1}{\epsilon}\left[\hat{L}(f)-\frac{e}{c}[\mathbf{v}, \mathbf{B}] \frac{\partial f}{\partial \mathbf{p}}\right] .
$$

Let us seek for the solution to equation (9) in a power series on the parameter $\in$ :

$$
f=f_{0}+\epsilon f_{1}+\ldots
$$

In zero approximation we have

$$
f(\mathbf{p}, \mathbf{r}, t)=f_{0}(|\mathbf{p}|, \mathbf{r}, t) .
$$


On the next step of this procedure, after averaging of equation (9) on the momentum direction, one can find:

$$
\frac{\partial f_{0}}{\partial t}+\epsilon \nabla\left\langle\mathbf{v} f_{1}\right\rangle+\epsilon\left\langle e \mathbf{E} \frac{\partial f_{1}}{\partial \mathbf{p}}\right\rangle=S_{0}\left(f_{0}\right)+S_{\text {ion }}\left(f_{0}\right)
$$

It is seen from (10), that the term $\frac{\partial f_{0}}{\partial t} \sim \epsilon$. Taking this. fact into account, we have in the first approximation on $\in$ :

$$
\hat{L}\left(f_{1}\right)-\frac{e}{c}[\mathbf{v}, \mathbf{B}] \frac{\partial f_{1}}{\partial \mathbf{p}}=\mathbf{p} \hat{\mathbf{H}} f_{0}
$$

here

$$
\hat{\mathbf{H}}=\frac{v}{p} \nabla+e \frac{\mathbf{E}}{p} \frac{\partial}{\partial p}
$$

It is naturally to seek for a solution to the equation (11) in a form:

$$
f_{1}=p_{i} A_{i k} \hat{H}_{k} f_{0}
$$

It is easy to check, that $f_{1}(12)$ is an eigenfunction of the operator $\hat{L}$,

$$
\hat{L} f_{1}=-\nu f_{1}
$$

That is why, the equation (11) is identical to another more simple equation 22

$$
-\nu f_{1}-\left[\mathbf{p}, \boldsymbol{\omega}_{B}\right] \frac{\partial f_{1}}{\partial \mathbf{p}}=\mathbf{p} \hat{\mathbf{H}} f_{0} \quad \omega_{B}=\frac{e B v}{c p} .
$$

As follows from equation (13) the matrix $A_{i k}$ has a form:

$$
\begin{aligned}
& A_{x x}=A_{y y}=-\frac{\nu}{\nu^{2}+\omega_{B}^{2}} \quad A_{z z}=-\frac{1}{\nu} \\
& A_{x z}=A_{z x}=A_{y z}=A_{z y}=0 \quad A_{x y}=-A_{y x}=-\frac{\omega_{B}}{\nu^{2}+\omega_{B}^{2}} .
\end{aligned}
$$

Substituting (12) into (10) we result in the equation for isotropic part of the distribution function $f_{0}$ :

$$
\begin{aligned}
& \frac{\partial f_{0}}{\partial t}+\frac{1}{3 p^{2}} \frac{\partial}{\partial p}\left[p^{2} e E_{i} A_{i k}\left(v \frac{\partial f_{0}}{\partial r_{k}}+e E_{k} \frac{\partial f_{0}}{\partial p}\right)\right] \\
& +\frac{\partial}{\partial r_{i}}\left[\frac{v}{3} A_{i k}\left(v \frac{\partial f_{0}}{\partial r_{k}}+e E_{k} \frac{\partial f_{0}}{\partial p}\right)\right]=S_{0}\left(f_{0}\right)+S_{\text {ion }}\left(f_{0}\right) .
\end{aligned}
$$

Equation (15) describes both thermal runaway effects and diffusive spreading of breakdown in the space. The analogous equation in nonrelativistic limit was derived in Ref. 22. If the magnetic field is negligible the distribution function in (15) is cylindrically symmetrical $f=f\left(t, p, r_{\perp}, z\right)$ and equation (15) could be rewritten in a form:

$$
\frac{\partial f}{\partial t}+I_{1}(f)+I_{2}(f)+I_{3}(f)=S_{0}(f)+S_{\text {ion }}(f)
$$


where

$$
\begin{aligned}
& I_{1}=\frac{1}{3 p^{2}} \frac{\partial}{\partial p}\left(p^{2} e^{2} E^{2} A_{z z} \frac{\partial f}{\partial p}\right) \\
& I_{2}=\frac{\partial}{\partial \mathrm{r}}\left(\frac{v^{2}}{3} A_{z z} \frac{\partial f}{\partial \mathrm{r}}\right) \\
& I_{3}=\frac{1}{3 p^{2}} \frac{\partial}{\partial p}\left(p^{2} v e E_{z} A_{z z} \frac{\partial f}{\partial z}\right)+\frac{\partial}{\partial z}\left(\frac{v}{3} e E A_{z z} \frac{\partial f}{\partial p}\right)
\end{aligned}
$$

The first term in (16), (17) describe the heating of electrons by electric field, the second one describe the diffusive spreading of breakdown in space and the third term describe the motion and acceleration of particles in discharge along electric field direction. The coefficient $1 / 3$ in (16), (17) appears because of scattering and angle averaging. Previously Ref. 6 was demonstrated that the breakdown discharge is spreading in space due to the scattering of a new born fast particles. Now we see that there is another process (angle scattering) which affect the diffusive spreading of breakdown discharge also.

Below, we restrict our consideration by a space uniform problem and neglect the dependence of $f$ on the variable $\mathbf{r}$. Equation (15) in these conditions has a form:

$$
\frac{\partial f_{0}}{\partial t}=\frac{1}{p^{2}} \frac{\partial}{\partial p}\left\{p^{2}\left[F_{D} f_{0}+\frac{e^{2} E^{2}}{3 \nu}\left(\frac{\nu^{2}+\omega_{B}^{2} \cos ^{2} \beta}{\nu^{2}+\omega_{B}^{2}}\right) \frac{\partial f_{0}}{\partial p}\right]\right\}+\hat{S}_{\mathrm{ion}}\left(f_{0}\right) .
$$

Here $\cos \beta=\frac{\mathrm{EB}}{E B}, \beta$ is an angle between electric and magnetic fields.

It is necessary to note that in spite of the equation (18) was derived in conditions of strong angle scattering of electrons in constant electric field, the quite analogous equation could be obtained in the case of oscillating or stochastic electric field. 22

\section{QUALITATIVE ANALYSIS}

We will begin from the analysis of the general kinetic equation (4). Since equation (4) is a linear one we can search for its solution in a form quite analogous to the one used in conventional breakdown theory ${ }^{23,24}$ :

$$
f(\mathbf{p}, t)=f(\mathbf{p}) e^{\lambda_{i} t}
$$

Here parameter $\lambda_{i}=\frac{1}{\tau_{i}}$ - is the ionization growth rate and $f(\mathbf{p})$ is a stationary distribution function which is established in the process of breakdown. It is determined by the stationary equation (4) with $\frac{\partial f}{\partial t}=\lambda_{i} f$.

Boundary conditions to this equation at the low energies are determined by the following physical process. Low energy electrons are generated by fast runaway electrons, they lose their energy in collisions and then disappear at very low energies due to dissociative or three body attachment of electrons to $\mathrm{O}_{2}$ molecules. That mean, that boundary conditions at $p \rightarrow 0$ is determined by the balance of the model ionization term $S_{\text {ion }}(f)$ and electron energy loss term $\hat{S}_{10}(f)$ :

$$
S_{\text {ion }}+\hat{S}_{0}(f)=0, \quad p \rightarrow 0
$$

As we will see below this condition determines quite definite mode of solution.

At the high energies when $p \rightarrow \infty$ it is natural to expect that:

$$
f \rightarrow 0, \quad p \rightarrow \infty
$$


Qualitatively the structure of the solution of stationary equation (4), (19) with boundary conditions (20), (21) could be understood basing on the parameters (7), (8) and the concrete form of distribution function for runaway problems obtained in previous works. ${ }^{19-22,25}$ At the low energy region $\gamma \leq 3-4$ (see (7)) distribution function is close to the spherically symmetric and gradually falls down with the energy of electrons $^{7} f \propto(\gamma-1)^{-1}$. At the high energies $\gamma>>8$ (see (8)) function $f(\mathbf{p})$ became directed along the direction of electric field (or in presence of a strong magnetic field - along the drift direction, analyzed in Ref. 13). Its directivity is high, but not too high determined by the combined action of electric field and ionization integral $S_{\text {ion }}(f)$. The distribution function can here effectively grow up with $\gamma$. It reaches maximum value at the second critical point $\gamma_{2 c}$ (see Fig. 1) and then rapidly falls down at $\gamma>\gamma_{2 c}$ analogous to Ref. 25.

It should be emphasized, that according to this analysis the range of energies were the stationary distribution function is determined is extremely large growing exponentially with $E / E_{c}$ (see Fig. 1). It means that in reality stationary distribution could be established at $E \sim E_{c}$ only. At $E \geq 2 E_{c}$ it is nonstationary and breakdown essentially depend on the process of developing and establishing of a stationary distribution function. The last process depends strongly on the form of initial distribution function $f_{0}(\gamma)$. In our case of the runaway breakdown in the atmosphere the initial distribution of fast electrons is determined by a cosmic ray secondaries, which have in most part the low energies $\varepsilon \leq(1-2) \mathrm{MeV}$. So we can consider:

$$
f_{0}=\left\{\begin{array}{ll}
F_{0}(\gamma), & \gamma \leq \gamma_{m} \\
0, & \gamma \geq \gamma_{m}
\end{array} \quad \gamma_{m} \approx 5\right.
$$

It will be shown below, that in this case the quasi stationary distribution function is rapidly established at $\gamma \leq \gamma_{m}$, but in the high energy range $\gamma>\gamma_{m}$ it is stretching out very slowly. It means in reality that breakdown process in initial conditions (22) is determined mainly by low energy electrons $\gamma \leq \gamma_{m}$. For this electrons condition (7) is well fulfilled and kinetic equation could be significantly simplified (16). Below we will use this equation to describe runaway air breakdown.

It should be notified also that as the full stationary solution is not established during runaway breakdown process in initial conditions (22), the electron distribution is stretching out with time in energies, what means that the number of electrons fast and slow is growing in time not strictly exponentially. This make it different from the classical breakdown process which is always growing exponentially in time. ${ }^{23,24}$

\section{RUNAWAY AIR BREAKDOWN}

Let us investigate now the problem of air breakdown, considering spherically symmetrical kinetic equation (18). Since equation (18) is a linear one, we can search for its solution in a form:

$$
f_{0}\left(\gamma, t^{\star}\right)=e^{\lambda_{i}^{\star} t^{\star}} f_{0}(\gamma)
$$

$\gamma$ and $t^{*}$ are nondimensional variables

$$
\gamma=\sqrt{\left(\frac{p}{m c}\right)^{2}+1}, \quad t^{\star}=\frac{t}{t_{0}}, \quad \lambda_{i}^{\star}=t_{0} \lambda_{i}, \quad t_{0}=\frac{m^{2} c^{3}}{4 \pi e^{4} Z n_{m} a}
$$

Substituting (23) in (18) and introducing parameters $\delta_{E}=E / E_{c_{0}}, h=B / E_{c_{0}}$ and $\delta_{z}=(1+Z / 2)^{-1}$, one can find from (18)

$$
\frac{d}{d \gamma}\left\{\left(\gamma^{2}-1\right) \phi_{D}(\gamma)\left[f_{0}+\frac{T_{\text {eff }}}{\gamma} \frac{d f_{0}}{d \gamma}\right]\right\}=\gamma \sqrt{\gamma^{2}-1} \lambda_{i}^{\star} f_{0}-S_{i}\left(f_{0}\right)
$$


Here $\gamma$ is Lorentz-factor of electron,

$$
\begin{aligned}
\phi_{D}= & \frac{1}{a} \frac{\gamma^{2}}{\gamma^{2}-1} \\
& \times\left\{8.4+\frac{1}{2} \ln \left(\gamma^{2}-1\right)+\frac{1}{2} \ln (\gamma-1)-0.347\left(\frac{2}{\gamma}-\frac{1}{\gamma^{2}}\right)+\frac{1}{2 \gamma^{2}}+\frac{(\gamma-1)^{2}}{16 \gamma^{2}}\right\}
\end{aligned}
$$

— is a friction force determined by interaction of electron with air molecules, and

$$
T_{\mathrm{eff}}=\frac{\delta_{E}^{2}\left(\nu^{2}+\omega_{B}^{2} \cos ^{2} \beta\right)}{3 \delta_{z}\left(\omega_{B}^{2}+\nu^{2}\right) \nu^{2} \gamma}
$$

- is a parameter which we will call effective temperature, $\omega_{B}=h / \gamma, \nu=\phi_{D} \delta_{z}^{-1}\left(\gamma^{2}-\right.$ 1) ${ }^{-1 / 2} \gamma^{-1}, a \approx 11.2$ - is a fundamental constant. ${ }^{6}$ Model ionization integral $S_{i}^{z}$ in (4) takes a form ${ }^{6,7}$ :

$$
S_{i}^{\prime}=\frac{1}{2 a} \int_{2(\gamma-1)+1}^{\infty} \gamma^{\prime 2} d \gamma^{\prime} f_{0}\left(\gamma^{\prime}\right)\left[\frac{1}{(\gamma-1)^{2}}-\frac{2 \gamma^{\prime}-1}{\gamma^{\prime 2}\left(\gamma^{\prime}-\gamma\right)(\gamma-1)}+\frac{1}{\left(\gamma^{\prime}-\gamma\right)^{2}}+\frac{1}{\gamma^{\prime 2}}\right]
$$

Note, that effective temperature $T_{\text {eff }}$ according to (26) depend significantly on the energy of electrons $\varepsilon=m c^{2}(\gamma-1)$. Thus, in the absence of magnetic field $(h=0)$ temperature $T_{\mathrm{eff}}$ in nonrelativistic limit $\gamma-1<1$ is proportional to $\varepsilon^{3}$. Directly this strong grows of $T_{\text {eff }}$ with $\varepsilon$ lead to thermal runaway effect.

The equation (4) has two asymptotics in the limit $\gamma \rightarrow 1$. They have different behavior at $\gamma \rightarrow 1$ : first one is $f_{0} \sim \exp \frac{1}{(\gamma-1)^{2}}$ and the second one $f_{0} \sim 1 /(\gamma-1)$. The first asymptotic corresponds to equipartition between two terms in LHS of the equation (4) and is equivalent to zero flux condition at $\gamma \rightarrow 1$. The second one is defined by the balance of friction force term in LHS and particle source $S\left(f_{0}\right)$ at RHS, it behaves as

$$
S\left(f_{0}\right) \propto \frac{1}{(\gamma-1)^{2}}, \quad f_{0} \propto \frac{1}{\gamma-1} .
$$

Only the second solution has a physical meaning in our case. Really, the fast particles from runaway region $\gamma-1>2 / \delta_{E}$ generate a wide range of electrons with low energies $\varepsilon$, which under the action of friction force lose their energy and disappear at $\varepsilon \rightarrow 0$ due to attachment to the air molecules. So the $\gamma \rightarrow 1$ asymptotic of distribution function is determined by the balance of particle generation and friction force, as was already noted in Section 2.

Integrating equation (25) over $\gamma$ we obtain the following expression for nondimensional ionization rate $\lambda_{i}^{*}(24)$ :

$$
\lambda_{i}^{\star}=\frac{\int_{1}^{\gamma_{\infty}}\left\{S_{i}(f)+\frac{d}{d \gamma}\left[\left(\gamma^{2}-1\right) \phi_{D} f\right]\right\} d \gamma}{\int_{1}^{\gamma_{\infty}} \gamma \sqrt{\gamma^{2}-1} f d \gamma}
$$

Here we took into account asymptotic behavior of distribution function at $\gamma \rightarrow 1$ (28). We supposed also in accordance with (21), (22) that $f \equiv 0$ at $\gamma \geq \gamma_{\infty}$. Note that close to the threshold conditions

$$
\lambda_{i} \rightarrow 0 \quad \text { at } \quad E=E_{t h}, \quad \text { or } \quad \delta_{E}=\delta_{t h}
$$

runaway breakdown process is always well described by the equations (18), (29) 
As it was shown in Section 2 the equation (18) strictly speaking has no exponential growth rate. To clarify this point let us consider the solution of equation (25) in a limit

$$
1 \ll \gamma \ll \gamma_{2 c}
$$

here $\gamma_{2 c}$ is a second root of equation

$$
e E=F_{D}(\gamma)
$$

(see Fig. 1) In this limit the equation (25) has following asymptotics the first one

$$
f^{(1)} \propto \gamma^{-\frac{3}{2}-\frac{1}{2} \sqrt{9+4 \lambda_{i} / \phi_{\infty} T_{\infty}}}
$$

and the second one

$$
f^{(2)} \propto \gamma^{-\frac{3}{2}+\frac{1}{2} \sqrt{9+4 \lambda_{i} / \phi_{\infty} T_{\infty}^{-}}}
$$

were

$$
\phi_{\infty}=\frac{8.5}{a} \quad T_{\infty}=\frac{\delta_{E}^{2} \delta z}{3 \phi_{\infty}^{2}}
$$

Numerical and analytical analysis show that the first asymptotic can not satisfy the boundary condition (28). The second asymptotic gives a growing up solution $f^{(2)}$ which contradict to the restriction (21). It means that in rigorous theory to obtain exponentially growing in time solution we should consider an extremely spread in energies solution of the full kinetic equation (4), taking into account directional part of distribution function analogous to Refs. 20, 25. But as it was shown earlier in real situation for runaway breakdown in atmosphere this solution is not realized.

Really, runaway breakdown arises only in the presence of a high energy electrons. In the air conditions these electrons are cosmic ray secondaries with the energy $\varepsilon \leq 1$ $\mathrm{MeV}$. So, the initial distribution function is not too spread in energies and could-be supposed as a beam which have an energy cut at $\varepsilon=\varepsilon_{m}$ (22).

Now, we will estimate the contribution of various parts of distribution function in growth rate (29). Let us suppose, that the main contribution is defined by the range of $\gamma \sim \gamma_{0}$ and $\gamma_{0}>1$. Taking into account (28) one can find from (29):

$$
\lambda_{i}^{*} \approx \frac{7}{4 a \gamma_{0}}
$$

So, we see, that contribution into the growth rate (31) decrease with increasing of $\gamma_{0}$. It means that the bulk part of distribution function $f(\gamma)$ for $\gamma \leq 1-2$ gives the main contribution into the $\lambda_{i}^{*}$.

Let us estimate now the spreading of the initial beam in time. The equation (18) is diffusive type in energy. For simplicity we restrict our consideration on relativistic limit. In this limit the simplified equation (18) takes a form:

$$
\frac{\partial f}{\partial t}=\frac{D}{\gamma^{2}} \frac{\partial}{\partial \gamma}\left(\gamma^{4} \frac{\partial f}{\partial \gamma}\right)+\delta(t) \delta\left(\gamma-\gamma_{m}\right) \quad D=T_{\infty} \phi_{D \infty} \approx 0.43 \delta_{E}^{2} \delta_{z}
$$

The solution of (32) has a following form:

$$
f \propto \frac{\exp \left\{-\frac{\left(\ln \gamma-\ln \gamma_{b}+3 D t\right)^{2}}{\sqrt{D t}}\right\}}{\sqrt{D t}}
$$


We see from (33) that the spreading of this beam and the motion of its maximum is rather slow (in limit $\delta_{z}<<1$ ). If the speed of maximum $D$ is lower, than $\lambda_{i}^{*}$,

$$
\lambda_{i}^{\star}>D
$$

we will have quasi-exponential growth rate. Using this condition we obtain from (32), (34) restrictions on critical field:

$$
\frac{E}{E_{c}}<1.5 \sqrt{\frac{\lambda_{i}^{\star}}{\delta_{z}}}
$$

It should be noted that $\lambda_{i}^{*}$ depend on $E / E_{c}$. This dependence was obtained previously ${ }^{6,7}$

$$
\lambda_{i}^{\star}=\lambda_{i 0}^{\star}\left(\frac{E}{E_{c}}\right)^{3 / 2} \quad \lambda_{i 0}^{\star} \approx \frac{1.6}{a}
$$

Using this relations one can find:

$$
\frac{E}{E_{c}}<3\left(\frac{1.6}{\alpha}\right)^{2}
$$

here $1 \leq \alpha \leq 1.3$ is a shielding $\operatorname{parameter}^{7}\left(Z_{\text {eff }}=Z / \alpha\right)$. If condition (36) is fulfilled the growth rate should be quasi-exponential.

We resume the results of analytical analysis in this section.

1. For atmospheric conditions the runaway air breakdown process could be described by simplified time dependent kinetic equation (18) with boundary conditions (28), (21) and initial distribution function (22).

2. The solution of equation (18) effectively depends on the form of initial distribution function and generally speaking does not grow exponentially in time. But for the initial conditions (22) and for not very high values of electric field (36) it could be close to exponential.

\section{NUMERICAL RESULTS}

Conventional air breakdown always grow in time exponentially. It is fully described by the threshold electric field $E_{c t h}$ and growth rate time $\tau_{i}$ which is rapidly diminishing function of $E / E_{c t h}$.

Runaway breakdown in general case is not growing exponentially in time. Its growth rate and threshold electric field depend significantly on the initial form of distribution function. Not far from the threshold runaway air breakdown process could be quite analogous to conventional, though it has much lower threshold electric field.

Here we will discuss the numerical solution of equation (18) with boundary conditions (21), (28) and a model ionization integral. First of all it is necessary to mention that numerical simulations justify analytical predictions about the behavior of the solution. We see (Figs. 2 and 3 ), that if the electric field is close to the threshold $\left(\delta_{t h} \approx 1.3\right.$ ) the electron density is growing up exponentially, but the exponent depends on the energy of initial beam $\varepsilon_{b}$.

5.1. Comparison with exact solution. Previously in the absence of magnetic field $B=0$ the solution of kinetic equation (4) with the model ionization integral $S_{\text {ion }}$ was investigated numerically. ${ }^{7}$ It is shown at Fig. 4 for $\delta_{E}=2$. One can see from the figure that the main bulk of electron distribution function is close to spherically 


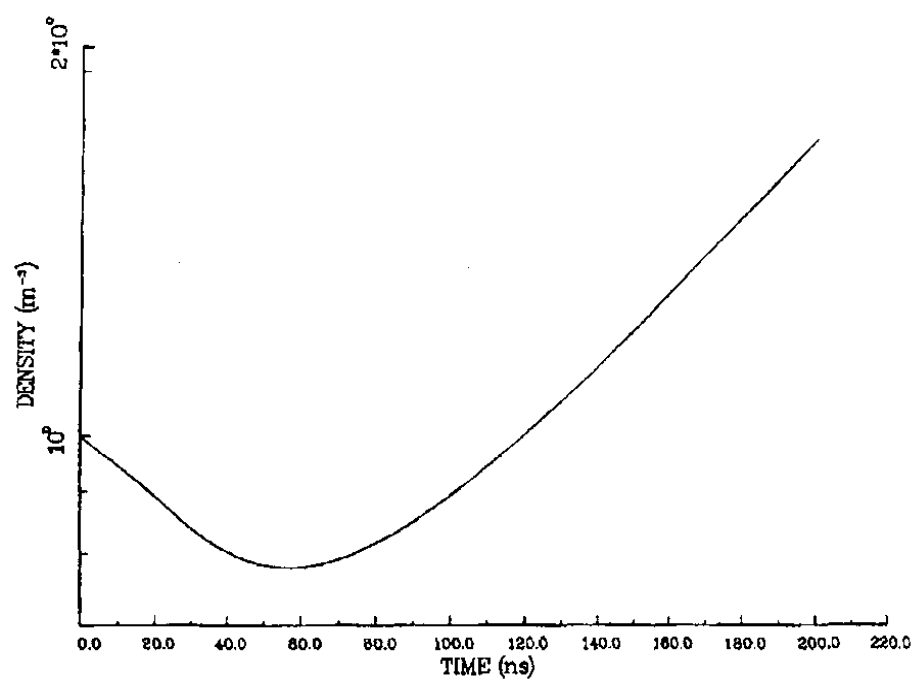

Figure 2. Spherically symmetric solution with $\varepsilon_{b}=10 \mathrm{MeV}$. The electron density is plotted as a function of time for $\delta_{E}=1.4, h=0$ and $\varepsilon_{b}=10 \mathrm{MeV}$.

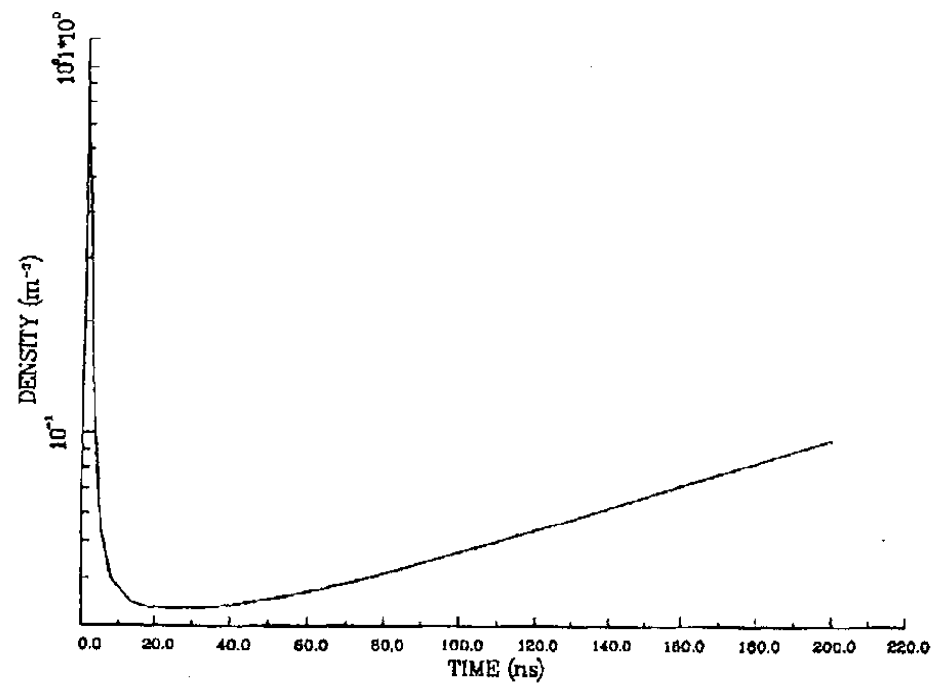

Figure 3. Spherically symmetric solution with $\varepsilon_{b}=1 \mathrm{MeV}$. The electron density is plotted as a function of time for $\delta_{E}=1.4, h=0$ and $\varepsilon_{b}=1 \mathrm{MeV}$. 


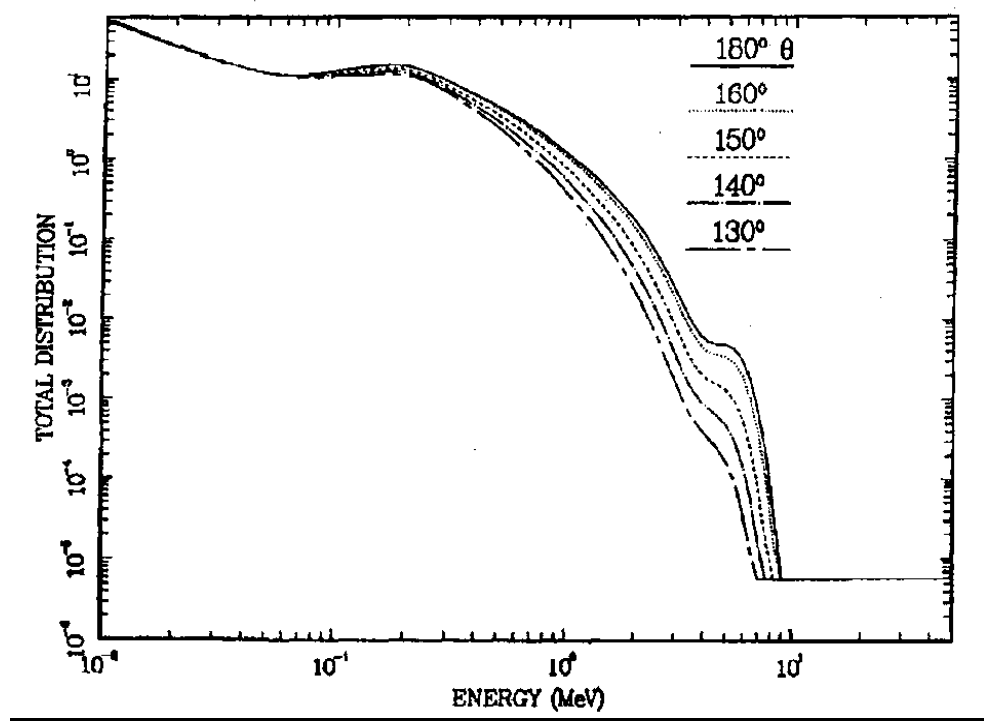

Figure 4. General solution of Boltzmann equation. The magnitude of the self-similar electron distribution function $\delta_{E}=2$ and no magnetic field $(h=0)$ is plotted as a function of the electron energy and the angle $\theta$ between the applied electric field and the electron momentum vector. These results were obtained in Ref. 7.

symmetrical. Quite analogous statement could be made about the form of distribution function $f$ for higher electric fields $\delta_{E}=5,8$, obtained in Ref. 7, though at the high energies $\varepsilon \geq 1 \mathrm{MeV}$ we see there, that the distribution function takes more directional character.

The solution of simplified spherically symmetrical equation (18) with boundary conditions (21), (28) and the same model ionization integral and initial distribution function as in Ref. 7 for $\delta_{E}=2$ is demonstrated at the Fig. 5. One can see quite a reasonable agreement with Fig. 4. The dependence of the growth rate $\lambda_{i}$ on electric field $\delta_{E}$ is presented in Table 1 . As we expected for the low value of $\delta_{E}$ the growth rate of electron density is close enough to the previous one and the difference between them is increasing with increasing of $\delta_{E}$.

The solution of equation (18) allowed to determine the threshold electric field for runaway breakdown with $B=0$, which was found to be $\delta_{t h} \approx 1.3$. We see from Figs. 4, 5 and Table 1, that not far from the threshold the form of distribution function and breakdown growth rate is well enough described in spherical symmetrical approach, what is in a full agreement with the results of analytical analysis, presented in the previous section. It allows us to explore the influence of magnetic field and oscillating electrical field on runaway breakdown in the same spherical symmetrical approximation.

5.2. Influence of magnetic field. The exact kinetic equation (4) is quite complicated for numerical analysis if magnetic field $\mathbf{B}$ is fully taken into account. The

Table 1. Avalanche Time for Spherical Symmetric $\tau_{i}^{s p}$ and Exact $\tau_{i}$ Kinetic Equa-

\begin{tabular}{lcc} 
tions & & \\
\hline$\delta_{0}$ & $\tau_{i}^{(s p)}(\mathrm{ns})$ & $\tau_{i}(\mathrm{~ns})$ \\
\hline 2 & 19.6 & 27 \\
5 & 2.4 & 7 \\
8 & 0.6 & 2.9 \\
\hline
\end{tabular}




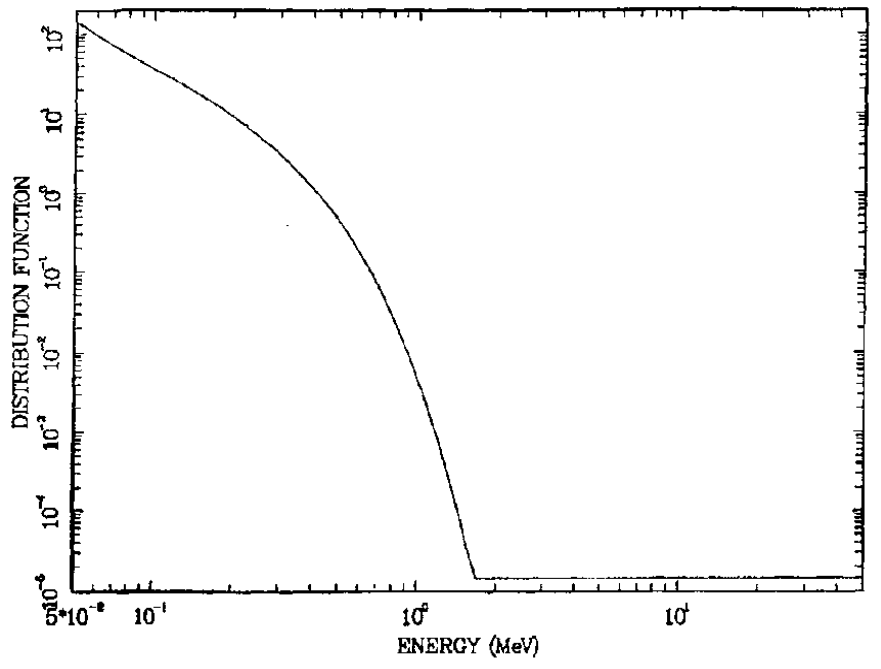

Figure 5. Spherically symmetric approach. The magnitude of the self-similar electron distribution function for $\delta_{E}=2$ and no magnetic field $(h=0)$ is plotted as a function of the electron energy. These results were obtained using the spherically symmetric approach and can be compared directly with the general solution obtained in Ref. 7 (see Fig. 4).

5.2. Influence of magnetic field. The exact kinetic equation (4) is quite complicated for numerical analysis if magnetic field $\mathbf{B}$ is fully taken into account. The above both analytical and numerical analysis shows that spherically symmetrical approach (18) is very useful if one consider solution not far from the threshold. Let us analyze now using this approach the dependence of the threshold electric field $\delta_{E_{t h}}$ on magnetic field B. It is characterized by two parameters: normalized value of magnetic field $h=B / E_{c 0}$ and angle $\beta$ between $\mathbf{B}$ and $\mathbf{E}$. The influence of magnetic field is mostly strong for $\beta=90^{\circ}$, when $\mathbf{E} \perp \mathbf{B}$. The dependence of $\delta_{E_{t h}}$ on $h$ for $\beta=90^{\circ}$ is shown on Fig. 6 and in Table 2. One can see from the figure and Table 2 that $\delta_{E_{t h}}$ is growing with $h$. This result seems quite natural: as one can see from equations (18), (26) the growth of magnetic field at $\beta=90^{\circ}$ diminishes the effective electron temperature $T_{\text {eff }}$. It means that the effective width of distribution function is decreasing with increasing magnetic field and one need to apply more strong electric field to achieve breakdown conditions. It is interesting to note that obtained in Ref. 13 analogous growth of breakdown electric field $\delta_{E_{t h}}$ with $h$ based on the analysis of the pure electron runaway process without heating, was much stronger. It means that the influence of thermal effects on runaway breakdown is enhancing with the increasing of normalized magnetic field $h$. This fact agrees with the form of distribution function in the presence of magnetic field obtained in Ref. 14 which is more close to the spherical symmetrical one (note that the amplification of breakdown time constant $\lambda_{i}$ with $h^{14}$ does not agree with our results).

The dependence of $\delta_{E_{t h}}$ on $h$ for different values of the angle $\beta$ are presented at Table 3. One can see that $\delta_{E_{t h}}$ for given $h$ is diminishing with diminishing $\beta$ and the relation

$$
\delta_{E_{t h}}\left(0^{\circ}\right)<\delta_{E_{t h}}(\beta)<\delta_{E_{t h}}\left(90^{\circ}\right), \quad \delta_{E_{t h}}\left(0^{\circ}\right) \approx 1.3
$$

Table 2. Threshold Electric Field for Runaway Breakdown with a Magnetic Field $\left(\beta=90^{\circ}\right)$

\begin{tabular}{lccccccc}
\hline$h$ & 0 & 1 & 2 & 5 & 10 & 30 & 100 \\
\hline$\delta_{t h}$ & 1.30 & 1.66 & 1.70 & 1.80 & 2.25 & 3.30 & 5.25 \\
\hline
\end{tabular}




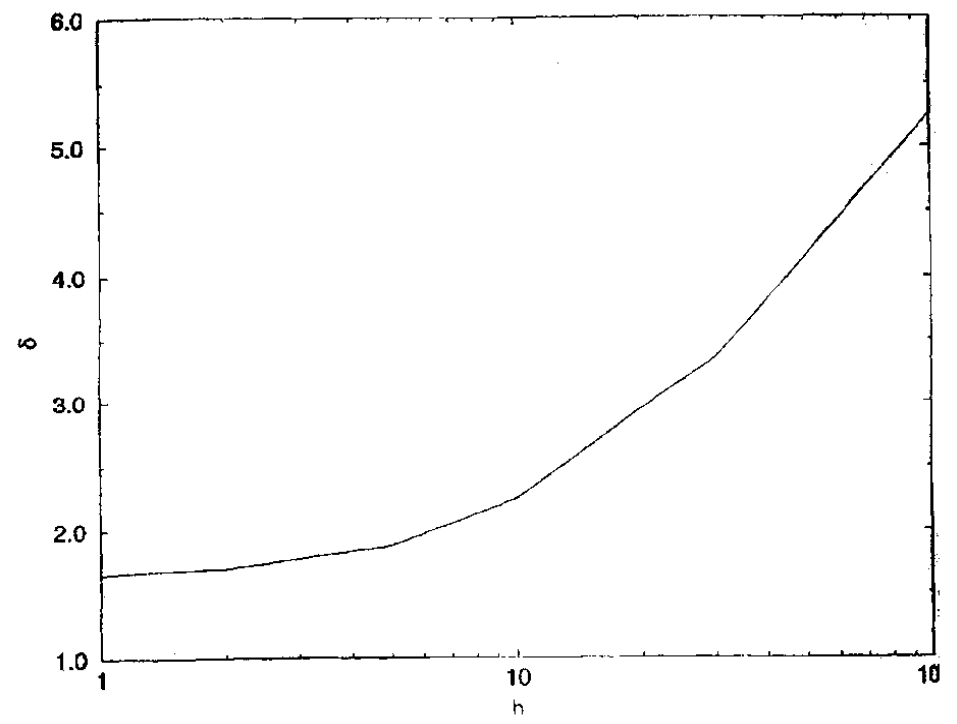

Figure 6. Threshold electric field vs. normalized magnetic field for $\beta=90^{\circ}$. The normalized threshold electric field for runaway breakdown $\left(\delta_{E_{t h}}\right)$ is plotted as a function of the normalized magnetic field (h) for an angle $\beta=90^{\circ}$ between the electric and magnetic fields.

is always fulfilled. This behavior of $\delta_{E_{t h}}$ naturally follows from the formulae for $T_{\text {eff }}$ in Eq. (18).

5.3. Alternating electric field. Spherical symmetrical approach and equation (18) gives the possibility to analyze breakdown in alternating and stochastic electric fields.

It is possible to show that in the case of stochastic field the equation (18) is not changed if one substitute averaged $\left\langle E^{2}\right\rangle$ instead $E^{2}$. Because of this all previous results for constant electric field are valid for stochastic field too.

The results of numerical solution of equation (18) for the case of alternating electric field are shown on Fig. 7 and 8. The solution in this case depends on relation between frequency of electric field $\omega$ and ionization rate $\lambda_{i}$. If

$$
\omega<\lambda_{i}
$$

the situation is quasistationary (see Fig. 7) and the total number of secondary electrons is following to the change of electric field oscillating with the frequency $\omega$. In opposite case when

$$
\omega>\lambda_{i}
$$

Table 3. Avalanche

Time as a Function of Angle $\beta$ for $\delta_{E}=2.0$,

\begin{tabular}{lc}
$\delta_{B}=10$ & \\
\hline$\beta($ degree $)$ & $\tau_{i}^{(s p)}(\mathrm{ns})$ \\
\hline 0 & 19.6 \\
15 & 72.1 \\
30 & 100 \\
45 & 273 \\
60 & $\infty$ \\
\hline
\end{tabular}




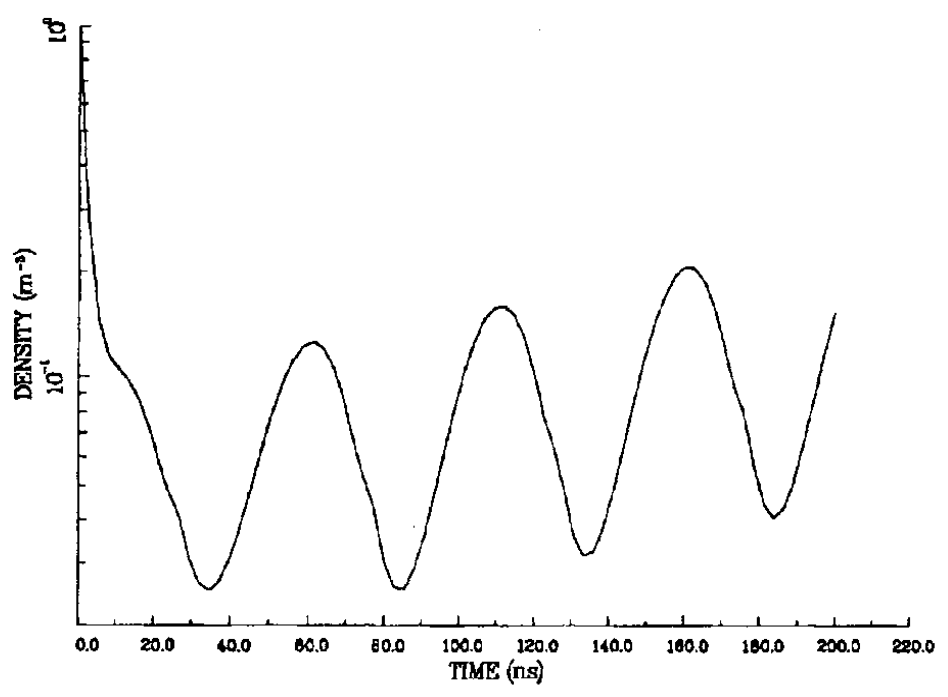

Figure 7. Oscillating electric field with $\omega<\lambda_{i}$. The electron density is plotted as a function of time for $\delta_{\omega t h}$ and $h=0$. In this case $\omega<\lambda_{i}$.

the breakdown is defined by the same equation (18), but the effective temperature takes the form ${ }^{22}$ :

$$
T_{\text {eff }}=\frac{e^{2} E_{0}^{2} \nu}{6\left(\nu^{2}+\omega^{2}\right)}
$$

Here $E_{0}$ - is the amplitude of oscillating electric field. We see that equation (40) if $\omega<<v$ coincide with the same equation for the constant electric field $E$, only this field is in $\sqrt{2}$ times less due to averaging of $E^{2}$ on oscillations. This averaging is clearly seen at Fig. 8. So the threshold amplitude for low frequency electric field should be

$$
E_{0 t h}=\sqrt{2} E_{t h}, \quad \delta_{\omega t h}=\sqrt{2} \delta_{t h}
$$

Numerical calculations confirm this results exactly giving $\delta_{\omega t h}=\sqrt{2} \delta_{t h} \approx 1.8$. Note, that in the nondimensional variables (4) frequency $\omega$ takes the form:

$$
h_{\omega}=\frac{m c \omega}{e E_{\mathrm{c} 0}}
$$

We see that the equation (18), (4) in normalized variables takes just the same form as in magnetic field with $h=h_{\omega}$. The only difference comes from the fact that $\omega_{B} \propto h / \gamma$, but $\omega$ does not depend on $\gamma$. form $^{22}$

In oscillatory electric field and magnetic field the effective temperature takes a

$$
T_{\text {eff }}=\frac{e^{2} E_{0}^{2}}{6}\left(\frac{\cos ^{2} \beta}{\nu}+\frac{\sin ^{2} \beta}{2\left[\left(\omega-\omega_{B}\right)^{2}+\nu^{2}\right]}+\frac{\sin ^{2} \beta}{2\left[\left(\omega+\omega_{B}\right)^{2}+\nu^{2}\right]}\right)
$$

We see, that in this case a gyroresonance effect is possible in conditions when $\beta=90^{\circ}$, $v$ is small enough $(h>>1)$ and frequency $\omega$ is close to $\omega_{B}$ at $\gamma \approx 1.3-1.5$.

\section{CONCLUSION}

Analytical and numerical arguments, presented in Sections 3-4 showed, that the kinetic equation for electrons describing runaway breakdown process in atmospheric 


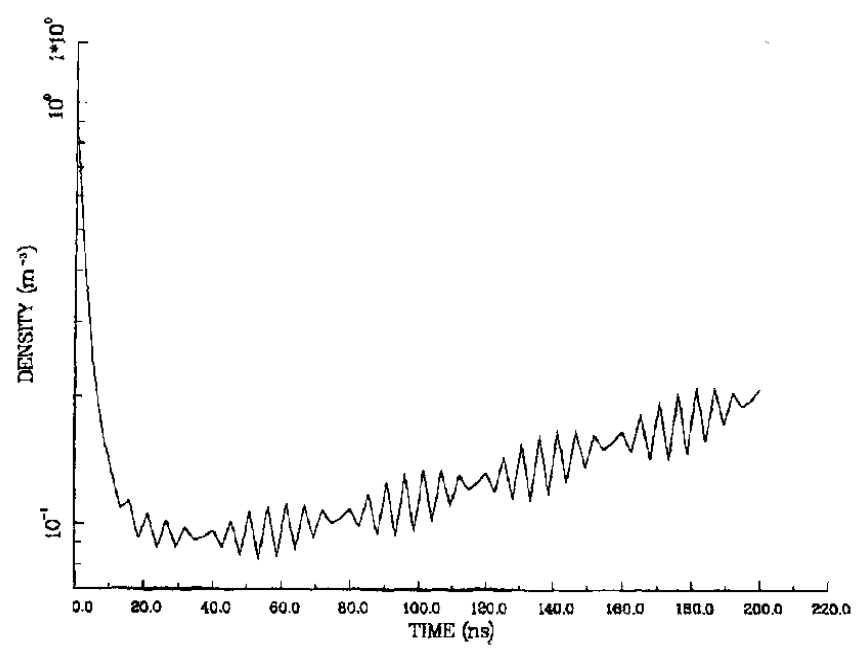

Figure 8. Oscillating electric field with $\omega>\lambda_{i}$. The electron density is plotted as a function of time for $\delta_{\omega t h}$ and $h=0$. In this case $\omega>\lambda_{i}$.

conditions could be significantly simplified and presented in spherical symmetrical form (18). It allowed us to solve this equation and determine breakdown threshold electric field and growth rate. In the first time this problem is consistently solved in the presence of the constant magnetic field, which plays a significant role in the Earth's atmosphere at the heights $z \geq 20-40 \mathrm{~km} .{ }^{13,14}$ In the Table 4 the threshold for a runaway breakdown by vertically directed electric field is presented as the function of height $z$ for northern $E_{t h}^{(n)} \quad\left(\beta \leq 30^{\circ}\right)$ and equatorial $E_{t h}^{(e q)}\left(\beta \approx 90^{\circ}\right)$ latitudes. Threshold electric field for the conventional breakdown $E_{c t h}$ is given in the Table also. One can see, that in the northern latitude (and midlatitudes) conditions runaway breakdown threshold fields is always much less than conventional

$$
E_{t h} \ll E_{c t h}
$$

But in equatorial region the same relation (44) is well fulfilled for the heights $z \leq 50$ $60 \mathrm{~km}$ only. This fact could be significant for understanding of the nature of high altitude lightning at different latitudes.

We have shown that runaway breakdown in homogeneous atmosphere can take place not only in a constant but in oscillating in time with any frequency electric fields also. The physical nature of this new effect is deeply connected with thermal runaway process. The possibility of existence of runaway breakdown in oscillating electric fields could be significant for interpretation of lower ionosphere ionization during thunderstorms ${ }^{26}$ and high altitude lightning mechanism. But the atmosphere at the heights $z \geq 50 \mathrm{~km}$ is strongly rarefied and due to this the spatial inhomogeneity could be significant here. So, a more detailed analysis of this problem is needed which lays out of the frame of present paper.

Table 4. Heights Dependence of Breakdown Parameters

\begin{tabular}{cccccc}
\hline $\mathrm{z}(\mathrm{km})$ & $N_{m} c m^{-3}$ & $E_{t h}^{(n)}(\mathrm{V} / \mathrm{cm})$ & $h^{(e q)}$ & $E_{t h}^{(e q)}(\mathrm{V} / \mathrm{cm})$ & $\mathrm{E}_{c t h}(\mathrm{~V} / \mathrm{cm})$ \\
\hline 0 & $2.7 \times 10^{19}$ & 2961 & 0.04 & 2900 & $2 \times 10^{4}$ \\
10 & $8.6 \times 10^{18}$ & 929 & 0.126 & 966 & $6.4 \times 10^{3}$ \\
20 & $1.8 \times 10^{18}$ & 195 & 0.60 & 226 & $1.33 \times 10^{3}$ \\
30 & $3.7 \times 10^{17}$ & 40.1 & 2.92 & 54 & 274 \\
40 & $8.2 \times 10^{16}$ & 8.9 & 13.2 & 17 & 60.7 \\
50 & $2.2 \times 10^{16}$ & 2.4 & 49.1 & 7.4 & 16.3 \\
60 & $6.7 \times 10^{15}$ & 0.72 & 161 & 3.7 & 4.9 \\
\hline
\end{tabular}




\section{ACKNOWLEDGMENT}

The authors express their appreciations to M. O. Ptitsyn and Yu. V. Medvedev for useful discussions and preliminary numerical calculations. The work was supported by ISTC grant $\mathrm{N} 480$.

\section{REFERENCES}

1. C. T. R. Wilson, Proc. Cambridge Philos. Soc. 22, 34 (1924

2. A. V. Gurevich, JETP, 39, 1296 (1960) [Sov. Phys. JETP bf 12, 904 (1961)].

3. G. E. Shaw, J. Geophys. Res. 72, 4623 (1967).

4. D. P. Whitmire, Lett. Novo Cim. 26, 497 (1979).

5. A. V. Gurevich, G. M. Milikh, R. A. Roussel-Dupreè, Phys. Lett. A 165, 463 (1992).

6. A. V. Gurevich, G. M. Milikh, R. A. Roussel-Dupreè, Phys. Lett. A 187, 197 (1994).

7. R. A. Roussel-Dupreè, A. V. Gurevich, T. Tunnel, G. M. Milikh, Phys. Rev. E 49, 2257 (1994).

8. M. P. McCarthy, G. K. Parks, Geophys. Res. Lett. 12, 393 (1985).

9. E. K. Eack, Rev. Sci. Instrum. 67, 2005 (1996).

10. E. K. Eack, W. H. Beasley, W. D. Rust, T. C. Marshal, M. Stolcenberg, J. Geophys. Res. (accepted for publication).

11. E. K. Eack, W. H. Beasley, W. D. Rust, T. C. Marshal, M. Stolcenberg, Geophys. Res. Lett. (1996) (accepted for publication).

12. G. J. Fishman, P. N. Bhat, R. Mallozzi et al., Science 264, 1313 (1994).

13. A. V. Gurevich, J. A. Valdivia, G. M. Milikh, K. Papadopoulas, Radio Sci. 31, 1541 (1996).

14. R. A. Roussel-Dupreè, R. H. Miller, EOS Trans. AGU 75, 44 (1994).

15. G. M. Milikh, K. Papadopoulas, C. L. Chang, Geophys. Res. Lett. 22, 85 (1995).

16. V. P. Pasko, K. S. Inan, Y. N. Taranenko, T. F. Bell, Geophys. Res. Lett. 22, 2127 (1995)

17. Y. N. Taranenko, K. S. Inan, T. F. Bell, Geophys. Res. Lett. 20, 1593 (1993).

18. Astrophysics of Cosmic Rays (ed. V. L. Ginzburg) Elsevier Science Publish. B. V. (1990).

19. A. V. Gurevich, JETP 38, 1597 (1960).

20. A. V. Gurevich, Y. N. Zhivlyuk, JETP 49, 214 (1965).

21. V. Y. Bychenkov, V. P. Silin, S. A. Uryupin, Physics of Plasmas, 10, 748 (1984).

22. A. V. Gurevich, Nonlinear Phenomena in the Ionosphere, Springer, NY (1978). .

23. A. D. MacDonald, Microwave Breakdown in Gases, NY-London-Sydney, Wiley (1966).

24. A. V. Gurevich, N. D. Borisov, G. M. Milikh, Physics of Microwave Discharges and Artificial Ionized Region in the Atmosphere, Gordon and Breach, Reading (1997).

25. A. V. Gurevich, JETP, 40, 1825 (1961) [Sov. Phys. JETP 13, 1282 (1961)].

26. U. S. Inan, J. V. Rodriguez, V. P. Idone, GRL 20, 2355 (1993). 